\title{
AGEING CHARACTERIZATION OF THE POWDER METALLURGY SUPERALLOY N18
}

\author{
Benjamin Flageolet, Patrick Villechaise, Mustapha Jouiad, José Mendez \\ Laboratoire de Mécanique et de Physique des Matériaux; Ecole Nationale Supérieure de Mécanique et d'Aérotechnique \\ 1 Avenue Clement Ader ; 86960 Futuroscope Chasseneuil ; France
}

Keywords: Ostwald ripening, $\gamma$ ' precipitates, ageing, $\gamma$ particles

\begin{abstract}
The ageing process of the powder metallurgy superalloy N18 has been investigated. This alloy exhibits a two-phase microstructure with a long range L12 ordered multimodal $\gamma^{\prime}$ precipitation.

N18 has been designed for high temperature turboengine disks applications. Superalloys however, are prone to microstructure evolution when sustained at high temperature since $\gamma^{\prime}$ precipitates are coarsening.

A method based on scanning electron microscope (SEM) image analysis is developed to qualify and quantify the material ageing. Two kinds of ageing have been considered: material temperature exposure with and without mechanical loading application. A short term quantitative law considering precipitates size, ageing time and temperature is established. In addition a long term behavior in agreement with our measurements is suggested.
\end{abstract}

\section{Introduction}

Turboengine developments have highly enhanced aircraft performances, however gas temperature at the turbine entry remains the key to increased engine efficiency. The material mechanical resistance at high temperature, specially against creep and fatigue clearly remains the technological barrier.

Nickel base superalloys have been developed and improved in the last three decades. These alloys are typically used in aeronautic industry for turbine blades and disks. They exhibit high mechanical properties in the temperature range 973-1373K. Because of numerous alloying elements (Al, Cr, Ti, Mo, W...) they combine high creep performance at high temperature and excellent oxidation and corrosion resistance. Their mechanical properties are due to the precipitation of a high volume fraction $(\sim 50-70 \%)$ of $\mathrm{Ni}_{3}(\mathrm{Al}, \mathrm{Ti})$ type precipitates $\left(\gamma^{\prime}\right)$, coherent with the $\gamma$ matrix.

The N18 alloy studied here is a polycrystalline powder metallurgy alloy designed for disks and elaborated by SNECMA Moteurs. This alloy is already used for military applications. In the framework of a program supported by the French research ministry this alloy is being evaluated for civil supersonic aircraft representative specifications. Such an application implies that the material used withstands fatigue loading at higher average temperature than military engines $\left(700-750^{\circ} \mathrm{C}\right)$.
Besides this, the temperature will be sustained for longer periods of time allowing nearly 30000 flights (instead of 3000 flights for military engines). In contrast, the effective stress amplitudes applied are supposed to be lower than the one in military aircrafts The evaluation of N18 microstructure evolution during long time temperature exposure and its consequences on mechanical properties and its creep and fatigue durability is one objective of this program. Several works have been performed on the ageing and rafting process of single crystal superalloys such as AM1 or CMSX1. These works have shown that high temperature dwell time leads to $\gamma^{\prime}$ phase coarsening and coalescence. However very few studies were focused on the ageing of polycrystalline alloys with multimodal $\gamma$ ' precipitation.

The aim of this study is to characterize the evolution of the fine $\gamma^{\prime}$ precipitation of N18 superalloy during long term temperature exposure. A special concern is given to the description of the phenomena involved in the ageing process considering various approaches issued from literature. An interpretation concerning the growth kinetic law of the $\gamma^{\prime}$ precipitation is proposed.

\section{Material}

The superalloy studied here is a polycrystalline powder metallurgy alloy. The nominal composition is given in Table 1[1]. N18 is hardened by the precipitation of $\gamma^{\prime}$ ordered L12 phase. The average grain size is around $15 \mu \mathrm{m}$. The standard thermal heat treatment applied to the alloy consists in a homogenisation of 4 hours at $1438 \mathrm{~K}$ followed by a $50-100 \mathrm{~K} / \mathrm{min}$ cooling step and two final annealing treatments ( 24 hours at $973 \mathrm{~K}+4$ hours at $1073 \mathrm{~K}$ ) leading to the precipitation of a large volume fraction of $\gamma^{\prime}$ particles within the austenitic $\gamma$ solid solution. The ordered $\gamma^{\prime}$ phase is consisted of 3 different scales of precipitation (Figure 1): -Primary $\left(\gamma_{\mathrm{I}}^{\prime}\right)$ precipitation along grain boundaries. The average precipitate size is $2-5 \mu \mathrm{m}$.

-Secondary $\left(\gamma_{\text {II }}^{\prime}\right)$ intragranular precipitation. The precipitates are mainly cubic and the mean edge length is $0.2 \mu \mathrm{m}$.

-Tertiary $\left(\gamma_{\text {III }}^{\prime}\right)$ intragranular precipitation. It is the finest population of precipitates. They are spherical and their diameter ranges from 0.02 to $0.05 \mu \mathrm{m}$. This population is known to highly influence creep properties.

\begin{tabular}{|c|c|c|c|c|c|c|c|c|c|c|c|}
\hline Element & $\mathrm{Ni}$ & $\mathrm{Cr}$ & $\mathrm{Al}$ & $\mathrm{Ti}$ & $\mathrm{Mo}$ & $\mathrm{Co}$ & $\mathrm{Hf}$ & $\mathrm{B}$ & $\mathrm{C}$ & $\mathrm{Zr}$ & $\mathrm{Fe}$ \\
\hline Atomic \% & 54.42 & 12.3 & 9.15 & 5.11 & 3.77 & 14.82 & 0.16 & 0.083 & 0.075 & 0.018 & 0.11 \\
\hline Weight \% & 57.05 & 11.4 & 4.41 & 4.37 & 6.47 & 15.6 & 0.52 & 0.016 & 0.016 & 0.03 & 0.11 \\
\hline
\end{tabular}

Table 1. N18 nominal composition 

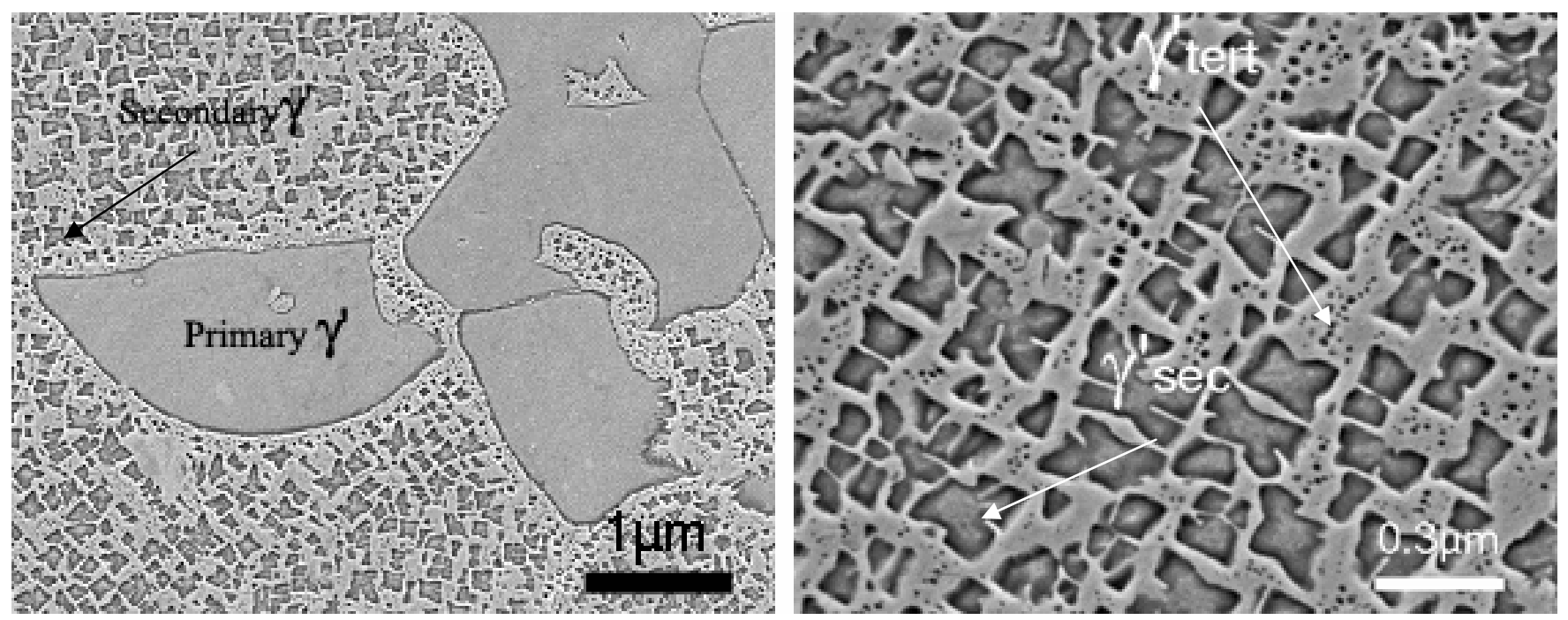

Figure 1. Standard microstructure

\section{Experimental procedures}

Accelerated ageing experiments were performed on standard N18 samples. They were conducted in a furnace at various temperatures ranging from $973 \mathrm{~K}$ to $1073 \mathrm{~K}$. In a first set of experiments, no mechanical loading was applied to the samples. They were only maintained in air at high temperature for a long period of time up to 600 hours. In a second set, some samples were aged under constant mechanical loading using a high temperature creep machine equipped with a high temperature furnace. Other specimens were aged under cyclic dynamic mechanical loading using a hydraulic fatigue machine.

All samples were then polished and etched in order to extract $\gamma_{\text {III }}^{\prime}$ precipitates to be observed in 6400 Jeol Scanning Electron Microscope (SEM) operating at $35 \mathrm{KV}$. For each ageing condition, ten to twenty images were taken and enhanced using Visilog 5.4 (Noesis) software. Typically, the number of particles measured for each condition is of the order of one thousand.

For Transmission Electron Microscopy (TEM), conventional 3 $\mathrm{mm}$ discs were cut using an electro spark machine. They were polished to a final thickness of about 70 micrometer by mechanical grinding using $\mathrm{SiC}$ papers. To be electron transparent, the samples were then prepared by jet polishing in a twin jet polisher Tenupol (Struers instruments). The electrolyte was a 10 $\%$ of perchloric acid in methanol solution. The experiments were performed on a Philips CM20 TEM operating at $200 \mathrm{kV}$.

\section{Results and discussion}

SEM and TEM observations show that $\gamma_{\text {III }}^{\prime}$ precipitates evolution is the main microstructure modification concerning $\gamma$ ' population evolution during high temperature dwell times. $\gamma^{\prime}{ }_{\text {II }}$ precipitates do not significantly evolve whereas $\gamma^{\prime}{ }_{\text {III }}$ particles become scarcer and increase their average diameter with ageing time. For this, the following investigations will be focused on $\gamma_{\text {III }}^{\text {phase evolution }}$ during ageing.

As a first approach, we assume that the $\gamma_{\text {III }}^{\prime}$ evolution is subjected to Ostwald ripening. This assumes that large precipitates grow at the expense of smaller ones owing to bulk diffusion phenomena. The whole process takes place in $\gamma$ matrix where precipitates put in common the $\gamma^{\prime}$ formers $(\mathrm{Al}, \mathrm{Ti} \ldots)$. The driving force is the Gibbs Thompson effect linking the precipitate radius to the local solute equilibrium concentration in the matrix.

The kinetic of this phenomenon has been widely studied for monomodal populations. The average precipitate size follows to a time dependent power law (equation 1). Basically, such process is described by the Lifshitz-Slyozov-Wagner theory (LSW) [2, 3] with a value of $m$ equal to $1 / 3$. LSW relation is given in equation 2 for bulk diffusion limiting process and for infinitesimal volume fraction of coarsening phase:

$$
\begin{gathered}
\bar{d}=\bar{d}_{0}+k t^{m} \\
d^{3}-d_{0}^{3}=\frac{64 D \sigma N_{\infty}}{9 R T} \times t=C t
\end{gathered}
$$

With D the solute efficient diffusion rate in the matrix, $\sigma$ the $\gamma / \gamma^{\prime}$ interfacial energy and $N_{\infty}$ the equilibrium solubility in the matrix $\gamma$. The linear evolution of $\mathrm{d}^{3}-\mathrm{d}_{0}{ }^{3}$ with the exposure time has been experimentally verified for several alloys[4-6].

Equation 2 has been modified taking into consideration non ideal solution [7].

$$
d^{3}-d_{0}^{3}=\frac{64 D V_{m}^{\gamma^{\prime}} \sigma}{9 G_{m}^{\gamma^{\prime \prime}} \times\left(C_{e}^{\gamma^{\prime}}-C_{e}^{\gamma}\right)^{2}} \times t
$$

Where $\mathrm{V}_{\mathrm{m}}$ is the molar volume of $\gamma^{\prime}$ phase, $G_{m}^{\gamma \prime}$ is the second derivative of the Gibbs energy of the matrix phase and $C_{e}^{\gamma^{\prime}}$ and $C_{e}^{\gamma}$ are the solute equilibrium solubilities in $\gamma$ and $\gamma^{\prime}$ phase.

Since real systems contain finite $\gamma$ ' volume fraction, equ 2 can be rewritten considering non zero volume fraction $[8,9]$ : 


$$
d^{3}-d_{0}^{3}=\frac{64 D V_{m}^{\gamma^{\prime}} \sigma K_{f v}}{9 G_{m}^{\gamma^{\prime \prime}} \times\left(C_{e}^{\gamma^{\prime}}-C_{e}^{\gamma}\right)^{2}} \times t=C t
$$

Where $\mathrm{K}_{\mathrm{fv}}$ is a monotonically increasing function of volume fraction. Despite numerous attempts to establish the effect of volume fraction on the coarsening kinetic, there is no clear effect of volume fraction either on the coarsening kinetic or the particle size according to Ardell [10]. Nevertheless, he notices that the elastic energy becomes stronger as the $\gamma^{\prime}$ volume fraction is increasing. Figure 2 illustrates experimental variation of the mean $\gamma_{\text {III }}^{\prime}$ "volume growth" of $\gamma_{\text {III }}^{\prime} d^{3}-d_{0}^{3}$ with the ageing time at $1058 \mathrm{~K}$.

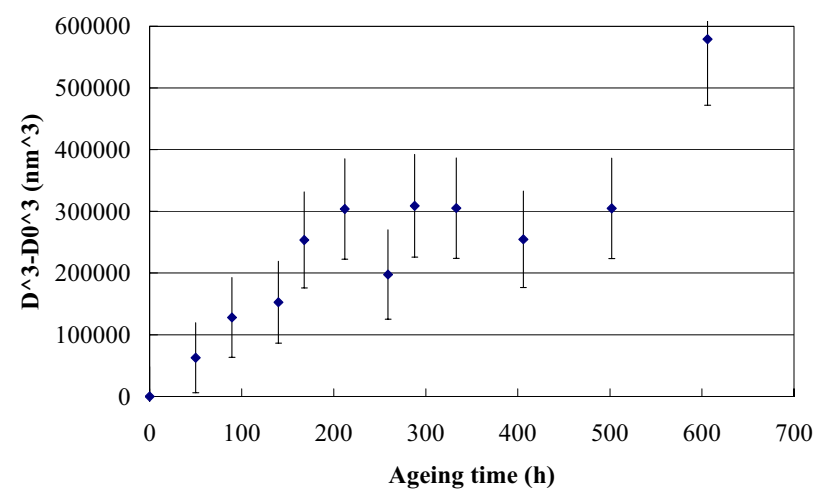

Figure 2. Experimental growth kinetic at $1058 \mathrm{~K}$

Below an ageing time of 200 hours, $d^{3}-d_{0}^{3}$ exhibits a linear evolution consistent with the LSW theory. Furthermore, in order to admit exponents different from 1/3, experimental measurements can be plotted according to equation 5

$$
\log \left(\bar{d}^{3}-\bar{d}_{0}^{3}\right)=A+3 m \cdot \log (t)
$$

Fitting experimental points up to an exposure time of about 200 hours (i.e in the linear range) with equation 4 , the exponent obtained is 0.35 . The coarsening process in the first stage of the ageing treatment applied in this study is then clearly following LSW theory.

In contrast, above 200 hours, the curve exhibits a plateau with a $\gamma_{\text {III }}^{\prime}$ mean size of $74 \mathrm{~nm}$ up to 500 hours ageing.

A typical set of population distribution obtained is provided in Figure 3. The particle size distribution (PSD) progressively changes from an initial narrow and small diameters-shifted distribution to a near Gaussian distribution for $333 \mathrm{~h}$. At the same time, the mean diameter increases from $48 \mathrm{~nm}$ to $74 \mathrm{~nm}$. However, above 333 hours and up to 600 hours, there is no evidence of PSD evolution and the mean diameter measured remains quasi constant. The $\gamma_{\text {III }}^{\prime}$ evolution is then not following LSW theory any more and another phenomenon is involved.

After 500 hours ageing, the particle growth is starting again and the average diameter is significantly increasing.

Several hypothesis can be discussed to explain the modification of the precipitate growth rate.
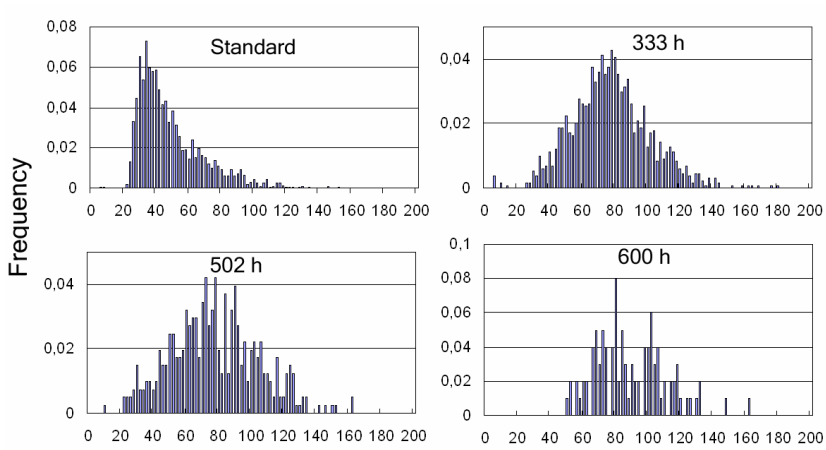

Diameter $(\mathrm{nm})$

\section{Figure 3. Particle size distribution for different ageing times}

-The etching method does not allow the extraction of the biggest precipitates.

-The coarsening process may lead to the coalescence of either tertiary precipitates population or tertiary and secondary particles.

-The elastic energy might become dominant regarding the surface energy, and lead to a decrease of the particle growth

-Some $\gamma$ ' formers may not be available any more for Ostwald ripening of tertiary precipitates because they would participate to the formation of other phases known as topologically closed packed phases (TCP) or carbides. These phases are observed in the case of long term ageing.

-Another phenomenon needing some $\gamma$ ' formers may be implied. The following sections detail arguments to discuss the relative pertinence of these different points.

\section{$\underline{\text { Influence of the etching procedure }}$}

In the case of an influence of the extraction method, it is clear that it would mainly concern the biggest particles, stucked between secondary precipitates. The particle size distribution would therefore be truncated. In fact the PSD corresponding to any ageing time belonging to the plateau are almost gaussian and the respective maximum diameter measured is well above the average value. After $600 \mathrm{~h}$ at $1058 \mathrm{~K}$, we can see the precipitate growth starting again. It is thus clear that the deviation from LSW law does not seem to be the consequence of the extraction procedure.

\section{Coalescence}

Two coalescence types are considered here.

-The first type corresponds to tertiary particles encountering secondary precipitates

-The second one is concerning coalescence between tertiary precipitates.

Tertiary particles absorption: In some specimens exposed $333 \mathrm{~h}$ at $1058 \mathrm{~K}$ and $400 \mathrm{~h}$ at $1023 \mathrm{~K}$, it has been observed (Figure 4) that some tertiary precipitates can encounter secondary ones. This phenomenon could be responsible for a decay in the particle growth estimation since the measures performed on aged specimens only concern tertiary precipitates. But it is worth noticing that such coalescence is only occurring in some scarce grains. Moreover, the evolution of secondary precipitates surface 
fraction during the ageing process does not reveal any increase as we could expect, but rather a very slight decrease. The coalescence effect can not explain the plateau in the $\gamma_{\text {III }}^{\prime}$ evolution. Furthermore, when specimens are aged up to 600 hours, particles are growing again.

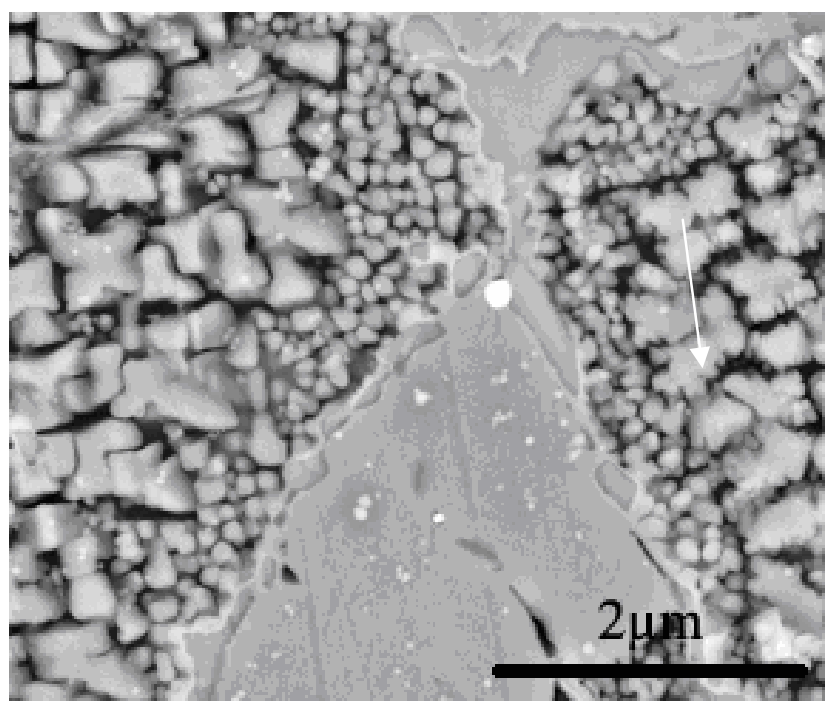

Figure 4. Coalescence with secondary precipitates

Tertiary particles encountering: Coalescence effects on the $\gamma_{\text {III }}$ size kinetic are not usually considered in coarsening theories. The phenomenon is rather rare. However, Davies et al. [11] modified LSW theory to take coalescence into account. According to these authors the expected effect of coalescence is an increased growth rate and a modification of the PSD which becomes broader and symmetrical during ageing. The $\mathrm{t}^{1 / 3}$ kinetic is still being observed. Sequeira [12], studying ageing in two different superalloys, observed different results with an exponent close to $1 / 2$ when coalescence occurs. A possible explanation for the disagreement of these observations with Davies' theory is that coalescence can not be considered as a bulk diffusion process. Indeed it is a rapid fusion after the encountering of two particles. Consequently it is obvious that massive coalescence could involve kinetic and PSD modifications. When two small precipitates encounter, they are eliminated from the PSD small size side while one appears in the large size side.

It is possible in the present study to know whether coalescence occurs or not during the ageing process by plotting and comparing the shape ratio distributions (Figure 5). This ratio is equal to 1 for a sphere and to $\sqrt{ } 2$ for a cube. It corresponds to the maximum dimension divided by the minimum dimension of a given particle. Distributions resulting from measures performed on N18 for ageing times ranging between 0 and 600 hours are gathered in Figure 5. The shape of the aspect ratio distribution remains stable during the whole process and centered on a value close to 1.3. Slight differences for long ageing time are not significant owing to the decreasing number of measured particles. No extra peak significant of important particle encountering and fusion can be seen and the width of the distributions is nearly constant. Thus there is no evidence of massive coalescence during the ageing treatment and the particles are almost spherical. According to our observations, it is not possible to conclude to an effect of coalescence on $\gamma_{\text {III }}^{\prime}$ coarsening kinetic.
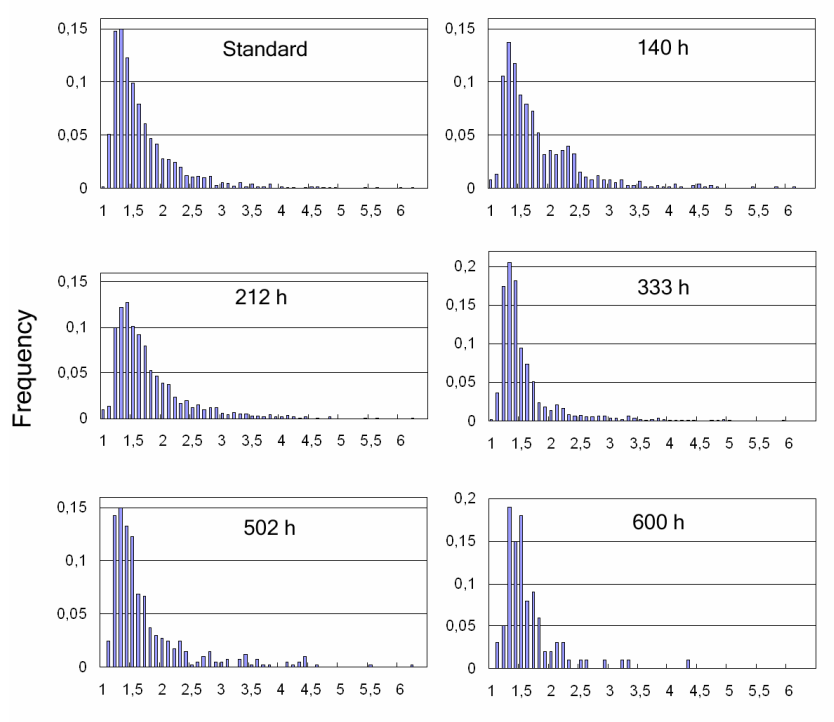

Aspect ratio

Figure 5. Aspect ratio distributions

\section{$\underline{\text { Elastic interactions }}$}

In the case of materials such as superalloys where precipitates are coherent with the matrix, the misfit can induce elastic interactions that become stronger with the increasing particle size. The different works performed on the effects of elastic interactions on the coarsening kinetic generally lead to the conclusion that elastic interactions become important only after long term ageing. Sources available in literature show that during the early steps of coarsening, the particle growth generally follows the LSW theory. Several authors [13-16] conclude the possibility of an inverted coalescence for the biggest particles (typically secondary particles) and a decreasing coarsening rate. Enomoto [16] mentione a new coarsening law following $\mathrm{t}^{1 / 2}$ for a negative misfit or an asymptotic decrease in the growth kinetic for positive misfit. Other authors [17] have observed in superalloys that the decrease in the coarsening is accompanied by morphological changes of particles, due to their size. Moshtaghin et al. [18] observed a decrease in the growth kinetic of the finest $\gamma^{\prime}$ particles in IN738LC, which they related to elastic interactions induced by temperature exposure. However their ageing experiments have been performed at $1123 \mathrm{~K}$ and for times up to $3000 \mathrm{~h}$. The range investigated here for N18 is more limited in terms of time and temperature. In our case, it is worth noticing that above $200 \mathrm{~h}$ ageing at $1058 \mathrm{~K}, \gamma_{\text {III }}^{\prime}$ growth is almost stopped. The change of the kinetic law from $\mathrm{t}^{1 / 3}$ to $\mathrm{t}^{1 / 2}$ or to an asymptotic decrease is consequently not a convincing explanation for the deviation from LSW in our observations. Furthermore, Leo in his model [19] mentioned a threshold precipitate size for the change in the kinetic growth of about $10^{6}$ times the size of the critical $\gamma^{\prime}$ phase precipitation radius. That is a threshold size of about $10^{-4} \mathrm{~m}$ for a nucleus of $1 \mathrm{~nm}$. Finally, no morphological changes or spatial correlation concerning tertiary particles have been shown, and the kinetic is starting again after $600 \mathrm{~h}$ at $1058 \mathrm{~K}$. The effects of elastic interactions are thus not evident in the present case. 


\section{Formation of TCP}

Using an appropriate etching method it is possible to reveal the presence of topologically closed packed phases along the grain boundaries as well as within the grains. Two main types of TCP are known to form during isothermal ageing in N18 alloy i.e $\mu$ phase and $\sigma$ phase[20]. Some carbides are also likely to form along grain boundaries. Analysing the formation kinetic during the ageing, a massive precipitation of needle like $\mu$ phase occurs after about 150 to 200 hours. Phases precipitating preferentially along grain boundaries start to form very early since they can be seen after 50 hours of ageing. These observations are consistent with the TTT diagram available for N18 alloy.

If the amount of tertiary particle (surface fraction) is measured at different steps of the ageing treatment, an important decrease is shown even in early stages. Wlodek et al. [21] obtained similar results and interpreted the reduction of the volume fraction of tertiary precipitates that they measured as a result of TCP precipitation. By means of X-ray diffraction led on previously extracted precipitates, he showed that rhomboedral $\mu$ phase as well as tetragonal $\sigma$ phase were $\mathrm{Co}, \mathrm{Mo}$, and $\mathrm{Cr}$ enriched. Since $\mathrm{Mo}$ and $\mathrm{Cr}$ can take place in $\mathrm{Al}$ sites and $\mathrm{Co}$ and $\mathrm{Cr}$ in $\mathrm{Ni}$ sites, Wlodek concludes that TCP precipitation is responsible for the dissolution of $\gamma^{\prime}$ III precipitates. In our case, it is obvious that the coarsening kinetic is almost stopped after $200 \mathrm{~h}$ of ageing and this would imply a $\gamma$ ' forming elements loss in the matrix. There is no significant amount of the $\gamma$ ' forming elements $\mathrm{Al}$ and Ti in TCP composition. As a consequence the deviation of $\gamma_{\text {III }}^{\prime}$ precipitate growth from LSW can not be attributed to TCP precipitation. Another phenomenon involving $\mathrm{Al}$ or $\mathrm{Ti}$ could occur in the alloy during isothermal ageing.

\section{$\gamma$ clusters formation and coarsening in $\gamma^{\prime}$}

Cadel et al. [1] observed the presence of $\mathrm{Cr}$ enriched particles within $\gamma^{\prime}$ phase in the alloy N18 using atom probe tomography. The total volume fraction of these particles measured in the material is $2,5 \%$ (4,3\% of the $\gamma^{\prime}$ volume). These particles are interpreted as $\gamma$ clusters formed during the air cooling step after the last heat treatment carried out at $1073 \mathrm{~K}$ during 4 hours. Analysing these particles with TEM the authors do not show order effects.

Field emission gun (FEG) SEM performed on the material presently studied show similar particles growing within primary $\gamma$ ' precipitates (Figure 6). Analysing them with TEM, there is no extra spot in the diffraction pattern and they seem to be coherent with the surrounding $\gamma^{\prime}$ phase (Figure 7).

The interpretation proposed by Cadel et al. is based on the high chromium content measured in $\gamma$ particles. The authors assume that they are the result of a chromium supersaturation occurring during air cooling from $1073 \mathrm{~K}$. Following this scenario, the $\mathrm{Cr}$ solubility would decrease with decreasing temperature whereas it would be the reverse for Al.

Because the material is air quenched from $1073 \mathrm{~K}$ and considering that diffusion is very low bellow $973 \mathrm{~K}, \gamma_{\text {I }}^{\prime}$ precipitates would not have enough time to stabilize. When specimens are exposed to a temperature lower than $1073 \mathrm{~K}, \gamma_{\mathrm{I}}^{\prime}$ would tend to stabilize by rejecting $\mathrm{Cr}$ and admitting more $\mathrm{Al}$ until the equilibrium solubilities would be reached. Considering this phenomenon from the matrix, it would lead to a loss in $\mathrm{Al}$ and to a break in $\gamma^{\prime}{ }_{\text {III }}$ growth kinetic. Once the aluminium amount necessary in $\gamma_{I}^{\prime}$ would be absorbed, then the coarsening process could restart.

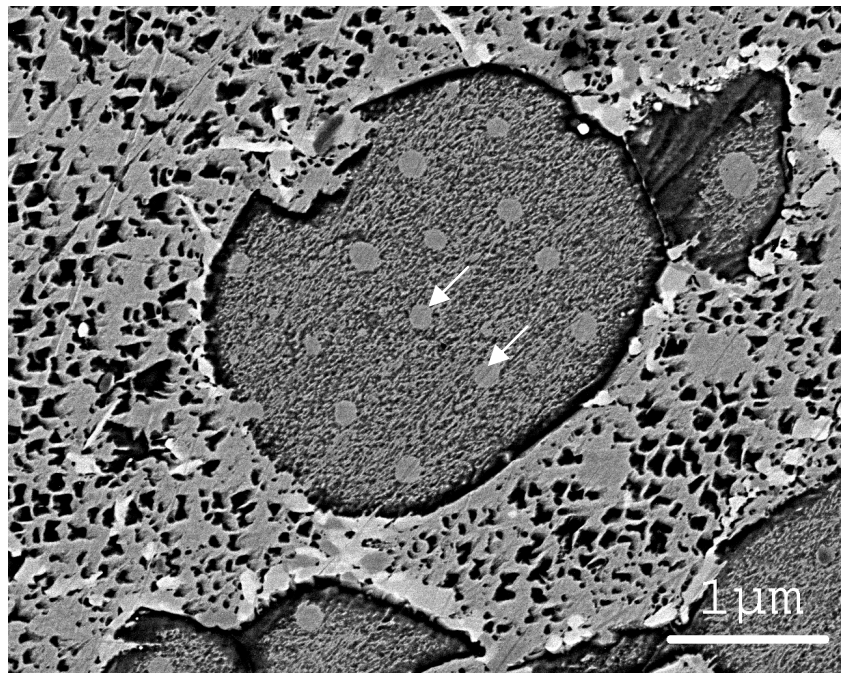

Figure 6. $\gamma$ particles (arrows) growing within primary precipitates after $300 \mathrm{~h} 785^{\circ} \mathrm{C}$ (FEG-SEM)

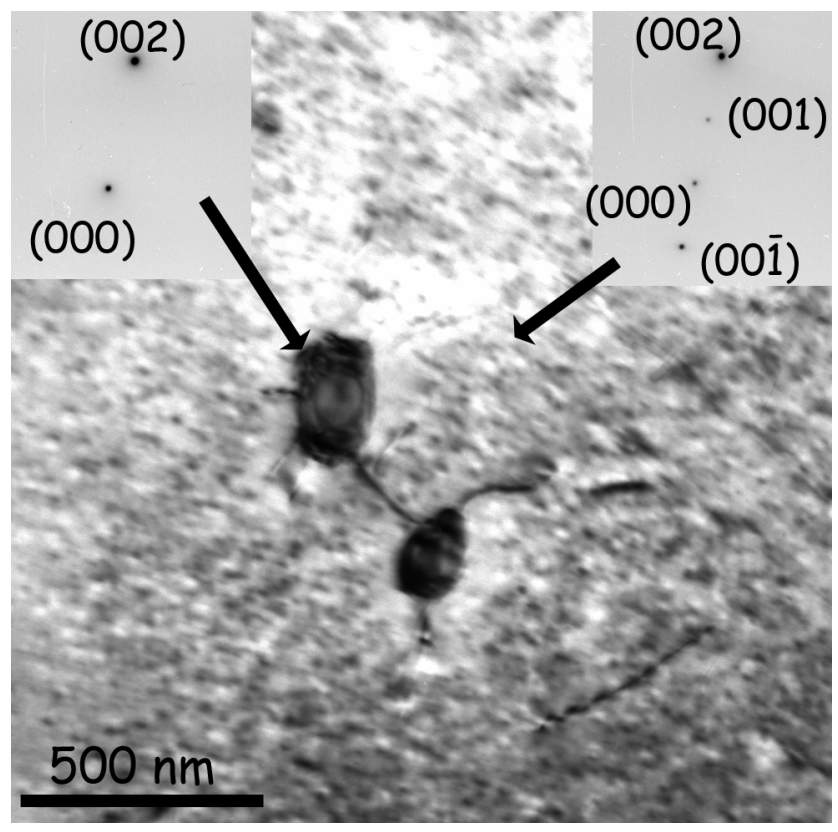

Figure 7. TEM image of $\gamma$ particle in a primary $\gamma^{\prime}$ precipitate

Considering that the absorption of $\gamma^{\prime}$ formers can be seen like the formation of some $\gamma^{\prime}$ particles with an average equivalent radius $\mathbf{r}_{\gamma}$, fluxes equality can then be rewritten as follows:

$>$ Flux feeding primary precipitates and the growth of tertiary precipitates

$$
F_{1}=\frac{4 \pi R^{2} D}{V_{m}} \frac{d N}{d R}
$$

Where $\mathrm{R}$ is the distance to the particle center, and $\mathrm{N}$ the solute molar fraction. 
$>$ Flux required for the growth of a tertiary particle:

$$
F_{t e r}=\frac{4 \pi r^{2}}{V_{m}} \frac{d r}{d t}
$$

Flux feeding an equivalent primary $\gamma$ ' precipitate:

$$
\begin{aligned}
& F_{\sigma}=\frac{4 \pi r_{\gamma}^{2}}{V_{m}} \frac{d r_{\gamma}}{d t} \\
& F_{1}=F_{t e r}+F_{\sigma}
\end{aligned}
$$

Integrating between $N_{\infty}$ and $N(r)$ and between $R=\infty$ and $R=r$

$$
D\left[N_{\infty}-N(r)\right]=\left[r^{2} \frac{d r}{d t}+r_{\gamma}^{2} \frac{d r_{\gamma}}{d t}\right] \times \frac{1}{r}
$$

Using the Gibbs Thompson law:

$$
\begin{array}{r}
N(r)=N_{\infty}\left[1+\frac{2 \sigma V_{m}}{R T r}\right] \text { we get: } \\
\frac{d r}{d t}=\frac{2 D V_{m} \sigma N_{\infty}}{R T r}\left(\frac{1}{r}-\frac{1}{r}\right)-\frac{1}{r}\left(r_{\gamma}^{2} \frac{d r_{\gamma}}{d t}\right)
\end{array}
$$

An approximate solution for the variation of $\bar{r}$ with time can be achieved by assuming that it will be the same as the maximum value of $\frac{d r}{d t}$

$\frac{d r}{d t}$ is maximum for:

$$
r=2 \bar{r}+\frac{R T_{\bar{r}}}{D V_{m} \sigma N_{\infty}}\left(r_{\gamma}^{2} \frac{d r_{\gamma}}{d t}\right)
$$

Using equ 12 in equ 11

$$
\frac{d \bar{r}}{d t}=\frac{\left(D V_{m} \sigma N_{\infty}\right)}{R \overline{T r}^{-2}\left(2 D V_{m} \sigma N_{\infty}+R \operatorname{Tr}_{\gamma}^{2} \frac{d r_{\gamma}}{d t}\right)}
$$

Assuming that $r_{\gamma}=k_{\gamma} t$ with $r_{\gamma}^{2} \frac{d r_{\gamma}}{d t}=k_{\gamma}^{3} t^{2}$

$$
\int_{0}^{r} r \bar{r} d \bar{r}=\frac{D V_{m} \sigma N_{\infty}}{2 R T} \int_{0}^{t} \frac{1}{1+\frac{R T k_{\gamma}^{3}}{2 D V_{m} \sigma N_{\infty}} t^{2}} d t
$$

$$
\begin{gathered}
\bar{r}^{3}-\bar{r}_{0}=\frac{3 D V_{m} \sigma N_{\infty}}{2 R T} \sqrt{\frac{2 D V_{m} \sigma N_{\infty}}{R T k_{\gamma}^{3}}} \arctan \left(\sqrt{\frac{R T k_{\gamma}^{3}}{2 D V_{m} \sigma N_{\infty}}} \times t\right) \\
\text { With diameter } \\
\bar{d}^{3}-\bar{d}_{0}^{3}=\frac{27 D V_{m} \sigma N_{\infty}}{2 R T} \sqrt{\frac{2 D V_{m} \sigma N_{\infty}}{R T k_{\gamma}^{3}}} \arctan \left(\sqrt{\frac{R T k_{\gamma}^{3}}{2 D V_{m} \sigma N_{\infty}}} \times t\right) \\
\bar{d}^{3}-\bar{d}_{0}^{3}=\frac{P_{1}}{P_{2}} \arctan P_{2} t
\end{gathered}
$$

For small ageing times, the kinetic growth is similar to LSW:

$$
\bar{d}^{3}-\bar{d}_{0}^{3}=P_{1} t
$$

Figure 8 shows the good correlation between equation 17 and experimental data. The absorption of Al by primary precipitates can therefore be considered as responsible for the kinetic slow down after $200 \mathrm{~h}$ ageing at $1058 \mathrm{~K}$.

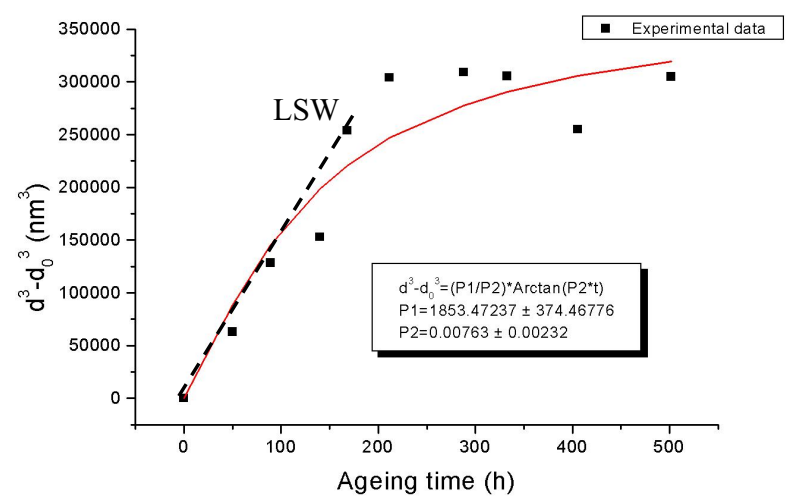

Figure 8. Experimental data fitted with (17)

\section{Temperature effect on the coarsening process}

If we limit our observations to the first stage of the ageing process i.e the part in agreement with LSW theory, it is possible to measure diffusion activation energy from kinetic curves at different ageing temperatures in the range 973-1073K. Specimens were aged and analyzed according to the procedure previously described. The measurements are reported in Figure 9.

The graph indicates that the growth rate of $\gamma_{\text {III }}$ increases with increasing temperature. 


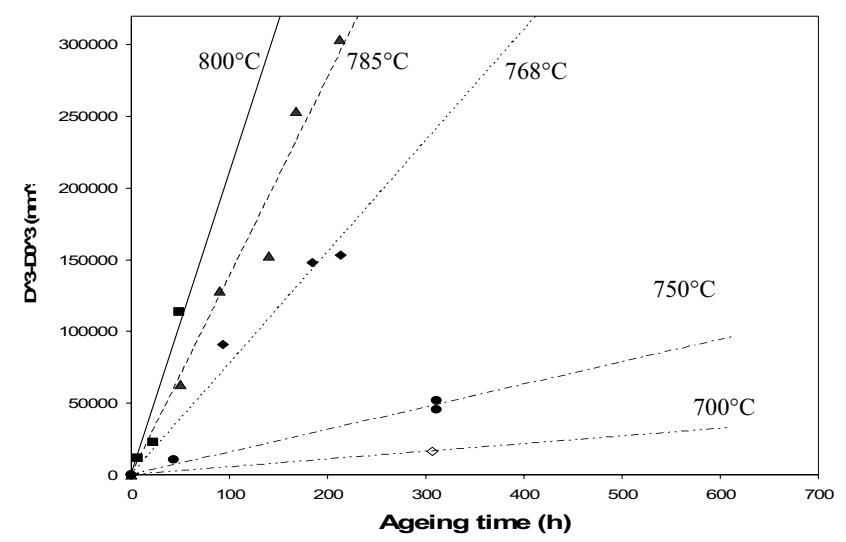

Figure 9. Temperature effect on the coarsening kinetic

Using the general law described by Calderon et al. for the growth kinetic in the linear domain, we develop the efficient diffusion coefficient in equation 3 :

$$
d^{3}-d_{0}^{3}=\frac{64 V_{m}^{\beta} \sigma D_{0}}{9 G_{m}^{\alpha^{\prime \prime}} \times\left(C_{e}^{\beta}-C_{e}^{\alpha}\right)^{2}} \times e^{\left(\frac{-Q}{R T}\right)} \times t=C t
$$

The efficient diffusion activation energy is calculated using the kinetic curves obtained for the five different ageing temperatures. Measuring the slope $\mathrm{C}$ for each temperature, we can then deduce (Figure 10) the activation energy $\mathrm{Q}$ by plotting $\operatorname{Ln}(\mathrm{C})=\mathrm{f}(1 / \mathrm{T})$ :

\section{$\mathrm{Q}=335 \pm 6 \mathrm{~kJ} / \mathrm{mol}$}

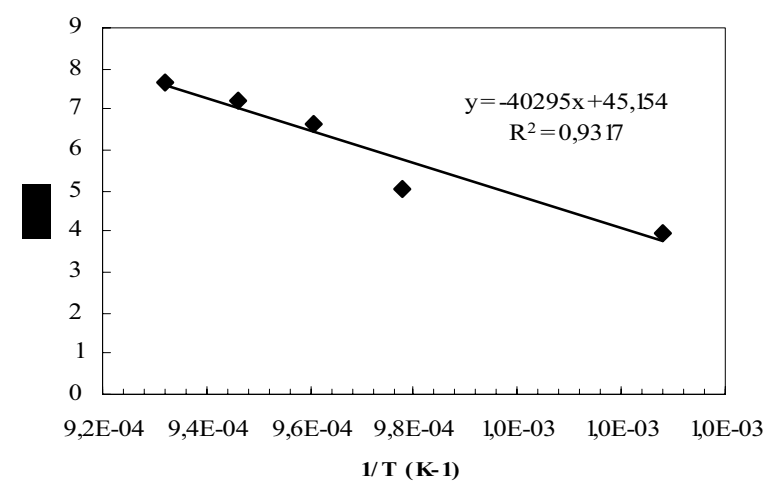

Figure 10. Measure of the effective activation energy

This value of the activation energy $\left(335 \mathrm{~kJ} \cdot \mathrm{mol}^{-1}\right)$ is to be compared to one reported in literature [22]: $260 \mathrm{~kJ} / \mathrm{mol}$ for the diffusion of $\mathrm{Al}$ in pure nickel. This difference is probably due to the fact that N18 contains numerous addition elements. Besides this, we can assess a value of this activation energy from internal friction works on N18 [23]: $340<\mathrm{Q}<410 \mathrm{~kJ} \cdot \mathrm{mol}^{-1}$.

Hence the experimental value calculated on linear domain of LSW theory is consistent with the rare values found in literature for the diffusion of aluminium in pure nickel $\left(260 \mathrm{~kJ} \cdot \mathrm{mol}^{-1}\right)$. Thus our approach to describe $\gamma^{\prime}$ III precipitate growth seems to be valid and we can write a size-time-temperature equivalence law in the linear growth domain using LSW theory.

Yet the influence of ageing temperature on the beginning and the length of the plateau is not clear. An interpretation according to the scenario previously exposed could be the following:

The time needed to reach the beginning of the plateau would correspond to the diffusion length necessary for Al to go from the matrix into primary precipitates. It would thus only be determined by the material microstructure and by the diffusion rate.

Considering the length of the plateau, it would depend on:

-Al solubility difference between $1073 \mathrm{~K}$ and the ageing temperature.

-The efficient diffusion rate

Hence if the ageing temperature is high (close to 1073K) the plateau would occur rapidly and would be short. In the opposite, if temperature is low then the plateau would begin after a long ageing time and would be long.

\section{Ageing under mechanical loading}

The method previously developed is consistent to describe the effect of time and temperature on precipitates coarsening, but it does not take into account the effect of mechanical loading. Therefore other experimentations have been performed in order to characterize its influence on the coarsening process. As it was previously précised, only the linear domain of the coarsening kinetic is considered. The result can then be compared to previous ones.

Two kinds of loading were applied on dog bone shaped specimens:

-Static loading: Samples were aged at $1023 \mathrm{~K}$ in a high temperature creep machine at two different stress levels: $200 \mathrm{MPa}$ and $300 \mathrm{MPa}$.

-Dynamic loading: Samples were aged at $1023 \mathrm{~K}$ in a servohydraulic fatigue machine. A triangular stress waveform was applied with a stress ratio equal to 0.05 . The stress amplitude is $800 \mathrm{MPa}$ and the frequency $0.5 \mathrm{~Hz}$.

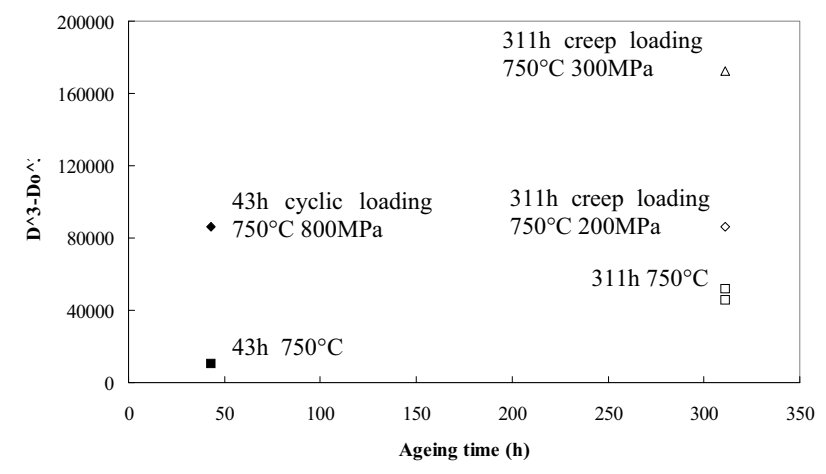

Figure 11. Effect of mechanical loading on the coarsening process

After ageing, the non loaded head of the specimens was analyzed and compared with the loaded part. Precipitates size measurements are realized using SEM observations and imaging software. The results obtained are plotted in Figure 11.

It is clear that static or dynamic loading enhance the coarsening rate. For static loading, the coarsening rate increases with increasing the stress applied. These observations are similar to the 
ones made by Skrotzki et al. [5] on age hardenable aluminium alloys. Al-Mg-Li samples exposed to $393 \mathrm{~K}$ under tensile creep loading (190-280MPa) show a higher growth rate than identical specimens aged without mechanical loading. Thus, it is worthy to include the stress parameter into the kinetic law to describe the ageing process of a turbine disk superalloy in near operating conditions. Further experiments are necessary at other temperatures to understand the way that stress influences the kinetic law.

\section{Conclusion}

In this study, we focused our attention on the evolution of tertiary precipitates population of the turbine disk superalloy N18. This population is prone to Ostwald ripening during high temperature exposure, and the average particle size is increasing while their number is decreasing during ageing. An original etching method is used to extract and measure precipitates. It allows size analysis on a high number of particles and leads to a significant estimation of the diffusion activation energy. Although it is not possible to get spatial distribution data since precipitates are not observed in their initial position in the matrix. However this method remains useful to provide average data for the study of the growth kinetic. According to our observations, the kinetic can be described in a given time-temperature range using a LSW type law based on a bulk diffusion controlled process. The value of the diffusion activation energy obtained from our measurements is typical for diffusion limited phenomenon.

Considering the $1058 \mathrm{~K}$ kinetic curve, a competition between tertiary $\gamma^{\prime}$ particles coarsening and $\mathrm{Al}$ absorption into primary $\gamma^{\prime}$ precipitates is proposed to interpret the appearance of a plateau in $\gamma_{\text {III }}^{\prime}$ growth kinetic.. It is thus necessary to take this competition into account to describe $\gamma^{\prime}$ III coarsening during the whole ageing process. A long term kinetic law in good agreement with experimental up to 500 hours is proposed.

Therefore, the understanding of the ageing process from initial stages up to an overaged microstructure using a time-temperature relation is essential to study the effect of ageing on mechanical properties such as creep and creep-fatigue durability.

The hypothesis concerning the effect of temperature on the position of the plateau is to be verified. Several experiments carried out at other temperatures than $1058 \mathrm{~K}$ and for longer ageing times are thus necessary.

The acceleration effect of mechanical loading in ageing is also important owing to our first experimental results. It is essential to take it into account in the kinetic law to improve the understanding of the creep-fatigue life reduction already observed Further experiments are necessary to qualify the loading effect and to clarify its influence in the case of realistic service conditions.

\section{Acknowledgements}

This work comes within the framework of a program financed by the French research ministry. It is developed in collaboration with other French laboratories (CEMES-Toulouse, and ONERA). The authors wish to thank SNECMA Moteurs for fruitful discussions and for providing the material used in this study.

\section{References}

1. E. Cadel D. Lemarchand S. Chambreland D. Blavette, "Atom probe tomography investigation of the microstructure of superalloys N18". Acta materialia, 2002. 50: p. 957-966.

2. I.M. Lifshitz V.V. Slyozov, "The kinetics of precipitation from supersaturated solid solutions". Journal of physical chemistery solids, 1961. 19(1/2): p. 35-50.

3. C. Wagner, "Theorie der Alterung von Niederschlägen durch Umlösen". Zeitschrift für Elektrochemie, 1961. Bd65(7/8): p. 581-591.

4. K. Mahalingam B.P. Gu G.L. Liedl T.H. Sanders, "Coarsening of d' (Al3Li) precipitates in binary Al-Li alloys". Acta metallurgica, 1987. 35(2): p. 483-498.

5. B. Skrotzki J. Murken. "On the effect of stress on nucleation, growth and coarsening of precipitates in agehardenable aluminium alloys". in Lightweight alloys for aerospace applications VI. 2001. Louisiana.

6. M. Heilmaier U. Leetz B. Reppich, "Order strenghtening in the cast nickel-based superalloy IN 100 at room temperature". Materials science and engineering, 2001. A319321: p. 375-378.

7. H.A. Calderon P.W. Voorhees J.L. Murray G. Kostorz, "Ostwald ripening in concentrated alloys". Acta metallurgica et materialia, 1994. 42(3): p. 991-1000.

8. A.J. Ardell, "The effect of volume fraction on particle coarsening : theoretical considerations". Acta metallurgica, 1972. 20: p. 61-71.

9. $\quad$ A.C. Lund P.W. Voorhees, "The effects of elastic stress on coarsening in the Ni-Al system". Acta materialia, 2002. 50: p. 2085-2098.

10. A.J. Ardell, "Observations on the effect of volume fraction on the coarsening of $g^{\prime}$ precipitates inbinary $\mathrm{Ni}-\mathrm{Al}$ alloys". Scripta Metallurgica, 1989. 24: p. 343.

11. C.K.L. Davies P. Nash R.N. Stevens, "The effect of volume fraction of precipitate on ostwald ripening". Acta metallurgica, 1979. 28: p. 179-189.

12. A.D. Sequeira H.A. Calderon G. Kostorz J.S. Pedersen, "Bimodal size distribution of gamma' precipitates in Ni-Al-Mo. Transmition electron microscopy". Acta metallurgica et materialia, 1995. 43(9): p. 3441-3451.

13. W.C. Johnson T.A. Abinandanan P.W. Voorhees, "The coarsening kinetic of two misfitting particules in an anisotropic crystal". Acta metallurgica, 1990. 38: p. 1349.

14. T.A. Abinandanan, "Coarsening of elastically interacting coherent particles". 1991, Carnegie Mellon University: Pittsburg. 
15. K. Kawasaki Y. Enomoto, "Statistical theory of ostwald ripening with elastic field interaction". Physica A, 1988. 150A: $\mathrm{p}$. 436.

16. Y. Enomoto K. Kawasaki, "Computer simulation of ostwald ripening with elastic interactions". Acta metallurgica, 1989. 37(5): p. 1399-1406.

17. P.K. Footner B.P. Richards, "Long term growth of superalloy $g^{\prime}$ particles". Journal of materials science, 1982. 17: p. 2141.

18. R.R. Moshtaghin S. Asgari, "Growth kinetics of $g^{\prime}$ precipitates in superalloy IN-738LC during long term aging". Materials and design, 2003. 24: p. 325-330.

19. P.H. Leo W.W. Mullins R.F. Sekerka J. Vinals, "Effect of elasticity on late stage coarsening". Acta metallurgica et materialia, 1990. 38(8): p. 1573.

20. C. Ducrocq A. Lasalmonie Y. Honnorat. "N18 a new damage tolerant PM superalloy for high temperature turbine disks". in Superalloys 1988. 1988.

21. S.T. Wlodek M. Kelly D. Alden. "The structure of N18". in Superalloys 1992. 1992. Cincinnati.

22. Y. Adda J. Philibert, "La diffusion dans les solides". Bibliothèque des sciences et techniques nucléaires ed. 1966.

23. P. Gadaud, Private communication. 2002, Laboratoire de Mécanique et de Physique des Matériaux: Poitiers (France). 
